\title{
The effects of tobacco smoking on age of onset of psychosis and psychotic symptoms in a first episode psychosis population
}

Authors: Lauren M. Hickling ${ }^{1}$; Victor Ortiz-García de la Foz ${ }^{2,3,4}$;Rosa Ayesa-Arriola ${ }^{2,3,4}$; Benedicto Crespo-Facorro ${ }^{2,3,4}$; Philip McGuire ${ }^{1}$; Rocio Perez-Iglesias ${ }^{1,4}$.

Affiliations: Department of Psychosis Studies, Institute of Psychiatry, Psychology and Neuroscience, King's College London, London, UK ${ }^{1}$; Marqués de Valdecilla University Hospital, IDIVAL Santander, Spain $^{2}$;Department of Psychiatry, School of Medicine, University of Cantabria, Santander, Spain ${ }^{3}$; CIBERSAM, Centro Investigación Biomédica en Red Salud Mental, Santander, Spain ${ }^{4}$.

Corresponding author: rocio.perez-iglesias@kcl.ac.uk

Running head: First episode psychosis, onset age \& smoking

Word count: 2898

Declaration of Interests: None

Key words: Psychosis, Age of onset, Smoking, Tobacco, First Episode Psychosis, Psychopathology

Background and Aims Research suggests that tobacco smokers may develop psychosis at an earlier age than non-smokers, with effects on psychotic symptoms. We aimed to test the difference in age of onset of psychosis between smokers and non-smokers. Design Self-report data was collected from smokers and non-smokers in a population of first episode psychosis patients. Setting Outpatient first episode psychosis programme in Santander (Cantabria), Spain. Participants Three hundred and ninety seven patients (226 male, 171 female) who agreed to take part between 2001 and 2011. Measurements Age of onset of psychosis, age of smoking initiation, demographics, family history of psychosis, and cannabis use were collected by self-report. Findings Kaplan-Meier analysis showed that smokers had a significantly lower mean age of psychosis onset (smokers=27.4 $( \pm 8.1)$ years, non-smokers $=30.5( \pm 9.9)$ years) than non-smokers $\left(\chi^{2}(1)=11.72, p=0.001\right)$. The Cox Proportional Hazard's Model showed no significant difference in the age of psychosis onset between smokers and non-smokers adjusted for covariates (hazard ratio $(H R)=1.034,95 \%$ Confidence interval (Cl) $0.828-1.291$ ). Age of psychosis onset was significantly predicted by cannabis use (HR=2.073, 95\% $\mathrm{Cl}$ 1.633-2.633) and gender ( $\mathrm{HR}=1.706,95 \% \mathrm{Cl}$ 1.363-2.135). Conclusions Smokers do not appear to have a significantly earlier age of psychosis onset than non-smokers after taking into account cannabis use and gender.

This article has been accepted for publication and undergone full peer review but has not been through the copyediting, typesetting, pagination and proofreading process which may lead to differences between this version and the Version of Record. Please cite this article as doi: 10.1111/add.13646

This article is protected by copyright. All rights reserved. 


\section{Introduction}

Tobacco smoking has been highly recognised as being associated with psychosis, due to its high prevalence rate in those with severe mental illness compared to the general population $(1,2)$. Increasing attention is being focused toward the suggestion that there is a relationship between tobacco smoking and onset of psychotic illness $(3,4)$.

Varied explanations have emerged as to why tobacco smoking is so prevalent in those with severe mental illness. Firstly, individuals may smoke to relieve the negative symptoms less affected by antipsychotics, an idea which has been supported by Jiang et al. (5). Secondly, to reduce medication side effects via the modulation of dopamine (6), and thirdly, to provide an anxiolytic effect (6).

A recent meta-analysis of 23 studies suggested that daily tobacco smokers developed psychosis an average of 1.38 years earlier than non-smokers (4).The authors commented that these results bring the self-medication hypothesis into question (4), as patients are smoking before onset of symptoms. Furthermore, the association between tobacco use and age at onset of psychosis has been found to persist despite controlling for confounders such as sex and cannabis use (7). Conversely, some research has suggested that smokers had a later age of onset than non-smokers (8), however in this study, 'smokers' were classified as people who smoked $\geq 7$ cigarettes a week in the past month, so this could include some sporadic smokers. In a meta-analysis of 29 studies, there was found to be no association between smokers and non-smokers in age at the onset of psychotic illness (9).

Research has also suggested that smoking tobacco may help alleviate some symptoms. Jiang et al. (5) found a significant improvement in negative psychotic symptoms in male patients with tobacco use. There was no effect of tobacco use on positive symptoms. Similar results have been found by other studies (10). Conversely, some studies have found no effect of smoking on either positive or negative symptoms in people with psychosis (11). However, a large study of patients with schizophrenia revealed a significantly higher Positive and Negative Syndrome Scale (PANSS) score in heavy smokers compared to mildly dependant smokers and non-smokers (12), as did Krishnadas et al. (13), who also found higher PANSS negative scores in those with mild-moderate dependence. Based on the findings of recent meta analyses $(4,9)$, we hypothesised that firstly smokers were more likely to have an earlier age of onset of psychosis than non-smokers. Secondly, due to the higher symptomatology scores seen in those with tobacco dependence in previous studies (13), it was hypothesised that smokers would have higher symptom scores than non-smokers at initial presentation. Thirdly, if tobacco use does have an impact upon psychiatric symptomatology we would expect a poorer treatment response in smokers, therefore it was hypothesised that smokers would have a poorer response to treatment than non-smokers.

\section{Methods}

\section{Design}

This article is protected by copyright. All rights reserved. 
Three hundred and ninety seven patients were assessed on various measures at baseline, with some outcomes also measured as week six, after a period of randomised antipsychotic treatment. A computer generated list was drawn up by a statistician, and patients were allocated by simple randomisation to receive either olanzapine $(5-20 \mathrm{mg} /$ day), risperidone $(3-6 \mathrm{mg} /$ day, haloperidol (3$9 \mathrm{mg} /$ day), quetiapine $(100-600 \mathrm{mg} /$ day), ziprasidone $(40-160 \mathrm{mg} /$ day) or aripiprazole $(5-30 \mathrm{mg} /$ day). All information was obtained at baseline, except for psychotic symptomatology, which was also measured at week six. This study is part of a clinical trial in first episode psychosis (Clinical Programme of First Episode Psychosis; PAFIP).

\section{Measures}

Tobacco cigarette use per day and the age at which the patient initiated smoking regularly (daily) was obtained in person by self-report. Patients who did not smoke were classified as 'non-smokers', those who smoked $<20$ a day were classified as 'smokers', and those who smoked $\geq 20$ a day were classified as 'heavy smokers'. Non-daily tobacco $(\mathrm{N}=2)$ and non-daily cannabis smokers $(\mathrm{N}=16)$ were classified as users, and former smokers were classified as non-smokers.

Duration of untreated illness (DUI) was defined as the time from the first unspecific psychosis symptoms (there should be no return to the previous stable level of functioning) to initiation of an adequate antipsychotic drug treatment. Duration of untreated psychosis (DUP) was defined as the time from the first continuous psychotic symptom present most of the time, to initiation of adequate antipsychotic treatment. Age of onset of psychosis was defined as the age at which the first continuous psychotic symptoms emerged.

Psychotic positive and negative symptoms were assessed with the SAPS and Scale for the Assessment of Negative Symptoms (SANS,(14)) respectively. Years of exposure to tobacco prior to illness was calculated by subtracting the age of initiation of tobacco use from age of onset of psychosis. SAPS and SANS measurements were conducted at baseline and after the first six weeks of pharmacological treatment.

Demographic information including sex was measured at baseline, cannabis use (yes/no), and the age at which this was started to be smoked regularly (daily) was also obtained in person by selfreport. Family history of psychosis was defined as having a first degree relative with a diagnosis of a psychotic illness (yes/no). These variables were chosen based on previous research in the area of tobacco use and the onset of psychosis $(15,16)$. Diagnoses were confirmed using the Structured Clinical Interview for DSM-IV (SCID-I) (17) carried out by an experienced psychiatrist. Our operational definition for a "first episode of psychosis" included individuals with a non-affective psychosis who have not received previously antipsychotic treatment regardless of the duration of psychosis. Symptom assessment was undertaken by the same assessor.

\section{Study Setting and Financial Support}

Patients were recruited from the inpatient and outpatient units at the University Hospital Marqués de Valdecilla, Santander (Cantabria), Spain. The site serves an epidemiologic catchment area population of 555,000 people. A more detailed description of this population has been previously published (18). 
Funding for the study was provided by the Mental Health Services of Cantabria. The study conformed to international standards for research ethics, and was approved by the local institutional ethics committee.

\section{Subjects}

Patients were eligible if they met the following criteria: 1) were aged 15 to 60 years, 2) were experiencing their first episode of psychosis, 3) were living in the catchment area, 4) met the DSM-IV criteria for a brief psychotic disorder, delusional disorder, schizophreniform disorder, schizophrenia, schizoaffective disorder or psychosis not otherwise specified, 5) had no prior treatment with antipsychotic medication, or if previously treated, a total lifetime of adequate antipsychotic treatment of less than 6 weeks, 6) were able to understand the aims of the study and signed an informed consent form, and 7) had current psychotic symptoms of moderate or greater severity assessed by one of the five items of the Scale for the Assessment of Positive Symptoms (SAPS, (19)). Patients were excluded for the following reasons: 1 ) they met DSM-IV criteria for drug dependence, 2) mental retardation, or 3) they had a history of neurologic disease or head injury. Between 20012011, 522 individuals were referred to the PAFIP first episode program. A final sample of 397 patients was considered for the present study (see Figure 1 for participant flow-chart).

\section{Figure 1: Participant flow-chart.}

\section{Statistical Methods}

Categorical variables were summarised as $n$ (percentage), and continuous variables as means and standard deviations (SD), baseline sociodemographic and clinical characteristics were tested with one-way-ANOVAs for continuous variables, or $\chi^{2}$ tests for the categorical variables.

Kaplan-Meier analysis was conducted on age of onset of psychosis, and smoking status (yes/no), and a second analysis on age of onset of psychosis and stratified smoking level (non-smoker, smoker and heavy smoker). The Cox Proportional Hazard's model was conducted to model the separate effects of gender, level of tobacco use, cannabis use, and family history of psychosis (yes/no) on age of onset of psychosis. A further analysis was conducted to model the effects of all these variables combined on age of onset of psychosis, with smoking type set as the categorical predictor. Main confounders (sex, cannabis use (yes/no), family history of psychosis (yes/no)) were entered as dichotomous covariates in the model. Non-smokers were considered to be the reference category. One-way ANCOVAs were used to compare smokers and non-smokers in terms of SAPS/SANS score at initial presentation, adjusting for sex, age, DUP, cannabis use (yes/no) and family history of psychosis (yes/no) . Finally, one-way ANCOVAs were used to compare smokers and non-smokers in terms of SAPS/SANS score at six weeks. Baseline score on SAPS/SANS were used as a covariate, along with sex, age, DUP, cannabis use (yes/no) and family history of psychosis (yes/no). Significance level was set $a$ priori at $\mathrm{p}<0.05$ and all methods were 2-tailed. Data was analysed using SPSS 21 (IBM Corp., Armonk, NY).

\section{Results}

Clinical and demographic characteristics of the sample are shown in Table 1. The mean age of the whole sample was $29.8( \pm 9.46)$ years, $56.9 \%$ were male, and $44.3 \%$ were unemployed. A family 
history of psychosis was reported in $33.4 \%$ of total patients, and the majority of patients had a diagnosis of schizophrenia (69\%). The mean age of onset of psychosis in all patients was $28.7( \pm 9.0)$ years old, and the mean age of initiation of daily smoking was $15.9( \pm 3.0)$ years. Therefore the patients who were smokers had been smoking for an average of $12.5( \pm 8.2)$ years before the onset of psychosis. All smokers initiated smoking before the onset of psychotic symptoms. Patients smoked an average of $19.35( \pm 9.2)$ cigarettes per day at baseline. The average DUI in all patients was $25.3( \pm 40.6)$ months and mean DUP was $13.8( \pm 30.8)$ months. At baseline, the mean SAPS score was $13.5( \pm 4.3)$ and mean SANS score was $7.1( \pm 6.3)$. Stratified by smoking status, patients did not differ in terms of total SAPS/SANS scores at baseline, DUI, age at smoking initiation or diagnosis (all p's $>0.05$ ), but significantly differed in sex, age, age at onset of psychosis, employment (yes/no), cannabis use (yes/no), and DUP (all p's <0.05). In the sample there were a total of 164 'nonsmokers', 80 'smokers' and 153 'heavy smokers'.

Table 1: Clinical and demographic characteristics of patients, stratified by level of tobacco use.

\section{Age of Onset of Psychosis}

Kaplan-Meier analysis showed that smokers had a significantly lower mean age of psychosis onset (smokers $=27.4( \pm 8.1)$ years and non-smokers $=30.5( \pm 9.9)$ years) than non-smokers $\left(\chi^{2}(1)=11.72\right.$, $p=0.001$, see Figure 2). When smoking status was stratified into level of smoking, there was a significant difference between the ages of psychosis onset in non-smokers (30.49 ( \pm 9.9$)$ years) and heavy smokers (27.2 $( \pm 7.6)$ years) $\left(\chi^{2}(1)=14.359, p<0.001\right)$.

Cox Proportional Hazard was conducted to assess the effect of smoking type on time to psychosis onset in years, adjusting for potential confounders. Non-smokers were the reference category, and smoking type was set as a categorical predictor (yes/no). The hazard ratio (HR) for smokers after taking into account sex, cannabis and family history was 1.034 times that of a non-smoker. This was not significant (see Figure 3). The results showed that cannabis use and sex had significant effects on age of onset of psychosis (see Table 2 for adjusted and unadjusted Cox Proportional Hazard results.

Figure 2: Kaplan Meier survival curve of smoking status and age at onset of psychosis. ${ }^{a}$

\section{Figure 3: Cumulative survival curve of smoking status and age at onset of psychosis. ${ }^{a}$}

Table 2: Adjusted and unadjusted hazard ratios for the association between age of onset of psychosis and tobacco use.

\section{Psychopathology and Treatment Response}

The ANCOVA analysis for SANS scores at baseline showed no significant difference between smokers and non-smokers when adjusting for covariates $(F(1,390)=1.226, p=0.269)$. The only significant independent predictor of SANS score at baseline was DUP $(F(1,390)=5.342, p=0.021)$. Similarly, there was no significant difference between smokers and non-smokers on SAPS score at baseline $(F(1,390)=0.142, p=0.706)$ when adjusting for covariates. There was however a significant relationship between SAPS score at baseline and cannabis use $(F(1,390)=4.375, p=0.037)$.

The ANCOVA analysis comparing change between SANS scores at baseline and at week- 6 , showed no significant difference between smokers and non-smokers when adjusting for covariates 
$(F(1,388)=1.112, p=0.292)$. Similarly, there was no significant difference between smokers and nonsmokers on SAPS score at week-6 $(F(1,388)=1.363, p=0.244)$ when adjusting for covariates.

\section{Discussion}

Initial Kaplan-Meier analysis demonstrated that smokers had a significantly earlier age of onset of psychosis when compared to non-smokers. However, after controlling for cannabis use, sex and a family history of psychosis, all well-known factors contributing to psychosis risk $(15,20)$, there was no effect of smoking status on the age of onset of psychosis. Variables that were significantly associated with an earlier age of onset of psychosis were sex and current use of cannabis.

The findings contradict those of Gurillo et al. (4), as there was found to be no effect of smoking tobacco on the age of onset of psychosis in the present study. Therefore, the results also go against the idea that smoking tobacco is associated with a later age of onset of psychosis as found by Ma et al. (8). However, in many studies investigating the effect of tobacco use on the age of psychosis onset, information regarding the use of substances such as cannabis is often unavailable (8), or limited (4). Gurillo et al. (4) found that the age of onset in smokers is significantly earlier than in nonsmokers ( 24.3 years vs 25.6 years respectively). The authors noted that because onset of smoking is earlier than symptom onset (ages of smoking initiation and psychosis onset not available), this brings the self-medication hypothesis into question. In the present sample, all patients started smoking (15.9 $( \pm 3.0)$ years) before the onset of their symptoms (27.4 ( \pm 8.1$)$ years), also bringing the selfmedication hypothesis into question. However, despite these findings, the overall risk of smokers versus non-smokers of developing psychosis at an earlier age was close to non-significance. This could explain the differing results between Gurillo et al. (4) and the present study. Furthermore Gurillo et al. (4) identified that the studies used in the meta-analysis comparing smokers versus nonsmokers were case-control studies, and when the analysis was restricted to only studies disclosing cigarette use per day the association between age of onset and tobacco use was no longer significant. Our findings support those of Myles et al. (8), as there was no significant difference in the age of onset of psychosis between smokers and non-smokers. The authors noted that the effect of tobacco use on psychosis age of onset may have been confounded by concurrent cannabis use in some patients, and furthermore, some of the studies included in analysis were different to those use by Gurillo et al. (4), which may account for their differing results.

Previous studies have observed less severe negative symptoms in smokers $(5,10)$. Jiang et al. (5) found that in males with acute schizophrenia, negative symptom scores were significantly lower in those that smoke $(H R=0.080, p=0.021)$ compared to non-smokers. Similarly, Smith et al. (10) found that smoking also led to lower mean negative symptom scores as measured by the PANSS (9.4 $( \pm 4.09)$ pre-test compared to $7.2( \pm 2.70)$ post-test) in inpatients diagnosed with schizophrenia or schizoaffective disorder. However in the present study there was no effect of smoking on either the positive or negative symptoms at initial presentation in patients with first episode psychosis.

Our results contradict the finding of Krishnadas et al. (12) and Aguilar et al. (11). Krishnadas et al. (12) interviewed 131 adults diagnosed with schizophrenia. The results showed significant differences between non-smokers, mildly dependent smokers and severely/highly dependent smokers in positive and negative symptom scores (13). Aguilar et al. (11) found that, in a sample of 250 outpatients diagnosed with schizophrenia, smokers had significantly higher scores on psychotic 
symptoms as measured by the PANSS compared to non-smokers. The findings of our study support those of Barnes et al. (11), who observed no significant difference in the positive or negative symptom scores in smokers and non-smokers. This sample was comprised of 146 outpatients and inpatients diagnosed with chronic schizophrenia, and was verified by expired carbon-monoxide levels (11).

Particular strengths of this study include the large sample size $(\mathrm{N}=397)$. Few studies have evaluated the relationship between smoking tobacco and the age of onset of psychosis, along with the effect of tobacco smoking on psychopathology of patients presenting with first episode psychosis. This study provides an accurate estimate of the effect of tobacco smoking on psychopathology and age of onset of psychosis, in patients presenting with their first episode, and is representative of first episode psychosis cases in this geographical area.

One key limitation of this study is that tobacco cigarette use was not biochemically verified. However research has shown that there is close comparability between self-reported and biochemical measures of tobacco smoking in people with psychosis (21). Another key limitation to this study is the potential for recall bias in recalling the age of initiation of smoking. It is also possible that the characteristics of the patients in the present study are not generalisable to those not presenting with their first episode of psychosis.

On the basis of these results, we can conclude that smoking tobacco has no effect on the age of onset of psychosis after taking into account cannabis use. Similarly, tobacco smoking also had no effect on psychopathology at initial presentation or treatment response at six weeks. Previous studies that have reported a relationship between tobacco and psychosis may be biased by cannabis use. Therefore, to be able to take into account the effect of smoking on psychosis age of onset and psychopathology we need to consider the use of cannabis in patients presenting with first episode psychosis.

\section{Acknowledgements}

We would like to that all the study participants for taking part in this study.

\section{References}

1. de Leon J, Diaz FJ. A meta-analysis of worldwise studies demonstrates an association between schizophrenia and tobacco smoking behaviours. Schizophr Res. 2005;76:135-57.

2. Lasser K, Boyd JW, Woolhandler S, Himmelstein DU, McCormick D, Bor DH. Smoking and Mental Illness: A Population-Based Prevalence Study. JAMA. 2000 Nov 22;284:2606-10.

3. Riala $K$, Hakko H, Isohanni $M$, Pouta $A$, Räsänen $P$. Is initiation of smoking associated with the prodromal phase of schizophrenia? Rev Psychiatr Neurosci. 2005;30:26-32.

4. Gurillo P, Juahar S, Murray RM, MacCabe JH. Does tobacco use cause psychosis? Systematic 
review and meta-analysis. Lancet Psychiatry. 2015;2:718-25.

5. Jiang J, See YM, Subramaniam M, Lee J. Investigation of Cigarette Smoking among Male Schizophrenia Patients. PLoS One. 2013;8:1-7.

6. Forchuk C, Norman R, Malla A, Martin M-L, McLean T, Cheng S, et al. Schizophrenia and the Motivation for Smoking. Perspect Psychiatr Care. 2002;38:41-9.

7. McGrath JJ, Alati R, Clavarino A, Williams GM, Bor W, Najman JM, et al. Age at first tobacco use and risk of subsequent psychosis-related outcomes: A birth cohort study. Aust New Zeal J Psychiatry. 2016;50.

8. Ma X, Li C, Meng H, Du L, Wang Q, Wang Y, et al. Premorbid tobacco smoking is associated with later age at onset in schizophrenia. Psychiatry Res. 2010;178:461-6.

9. Myles N, Newall H, Compton MT, Curtis J, Nielssen O, Large M. The age at onset of psychosis and tobacco use: a systematic meta-analysis. Psychiatr Epidemiol. 2012;47:1243-50.

10. Smith RC, Infante M, Ali A, Nigam S, Kotsaftis A. Effects of Cigarette Smoking on Psychopathology Scores in Patients With Schizophrenia: An Experimental Study. Subst Abus. 2001;22:175-86.

11. Barnes M, Lawford BR, Burton SC, Heslop KR, Noble EP, Hausdorf K, et al. Smoking and schizophrenia: is symptom profile related to smoking and which antipsychotic medication is of benefit in reducing cigarette use? Aust N Z J Psychiatry. 2006;40:575-80.

12. Aguilar MC, Gurpegui M, Diaz FJ, de Leon J. Nicotine dependence and symptoms in schizophrenia: naturalistic study of complex interactions. Br J Psychiatry. 2005;186:215-21.

13. Krishnadas R, Jauhar S, Telfer S, Shivashankar S, McCreadie RG. Nicotine dependence and illness severity in schizophrenia. Br J Psychiatry. 2012;201:306-12.

14. Andreasen NC. Scale for the Assessment of Negative Symptoms. lowa City, la: University of lowa; 1983.

15. Schlosser D a., Pearson R, Perez VB, Loewy RL. Environmental Risk and Protective Factors and Their Influence on the Emergence of Psychosis. Adolesc Psychiatry (Hilversum). 2012;2:16371.

16. Stefanis NC, Dragovic M, Power BD, Jablensky A, Castle D, Morgan V a. The effect of drug use on the age at onset of psychotic disorders in an Australian cohort. Schizophr Res. Elsevier B.V.; 2014;156:211-6. 
17. First MB, Spitzer RL, Gibbon M, Williams JBW. Structured Clinical Interview for DSM-IV Axis I Disorders. Washington D.C.: American Psychiatric Press Inc.; 1996.

18. Pelayo-Terán JM, Pérez-Iglesias R, Ramírez-Bonilla ML, González-Blanch C, Martínez-García O, Pardo-García G, et al. Epidemiological factors associated with treated incidence of firstepisode non-affective psychosis in Cantabria: Insights from the Clinical Programme on Early Phases of Psychosis. Early Interv Psychiatry. 2008;2:178-87.

19. Andreasen NC. Scale for the Assessment of Positive Symptoms. lowa City, la: University of lowa; 1984.

20. McGrath J, Saha S, Welham J, El Saadi O, MacCauley C, Chant D. A systematic review of the incidence of schizophrenia: the distribution of rates and the influence of sex, urbanicity, migrant status and methodology. BMC Med. 2004;2:13.

21. Tidey JW, Rohsenow DJ, Kaplan GB, Swift RM, Adolfo AB. Effects of smoking abstinence, smoking cues and nicotine replacement in smokers with schizophrenia and controls. Nicotine Tob Res. 2008;10:1047-56. 
Figure 1: Participant flow-chart.

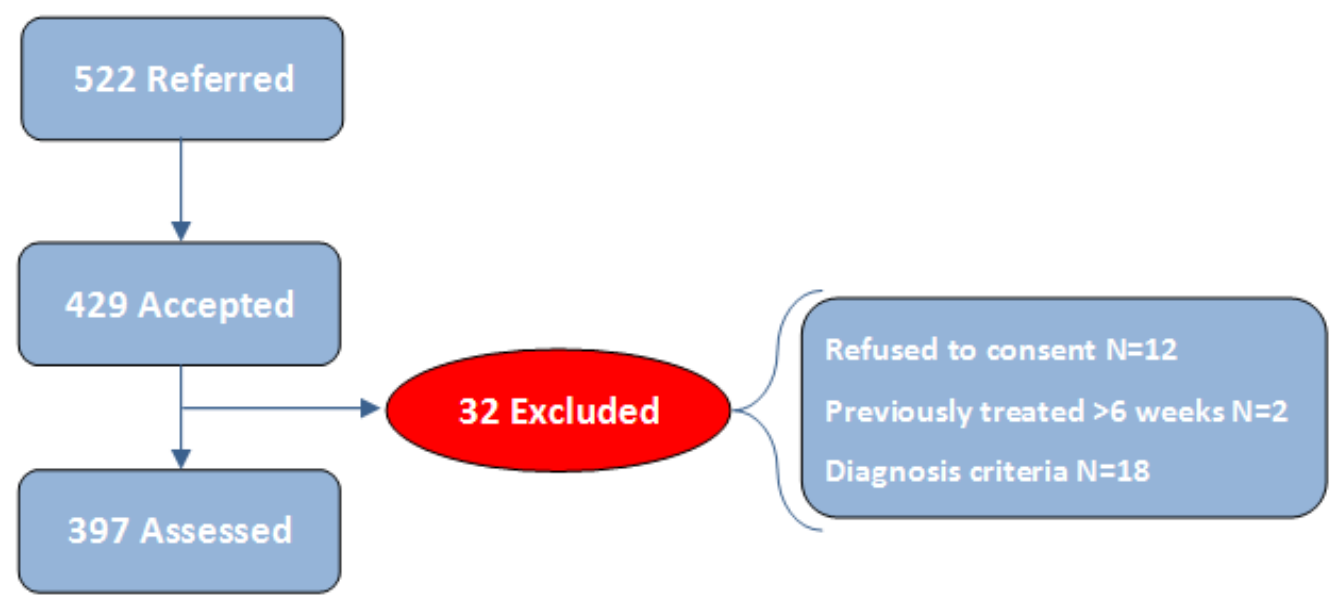

This article is protected by copyright. All rights reserved. 
Figure 2: Kaplan Meier survival curve of smoking status and age at onset of psychosis. ${ }^{a}$

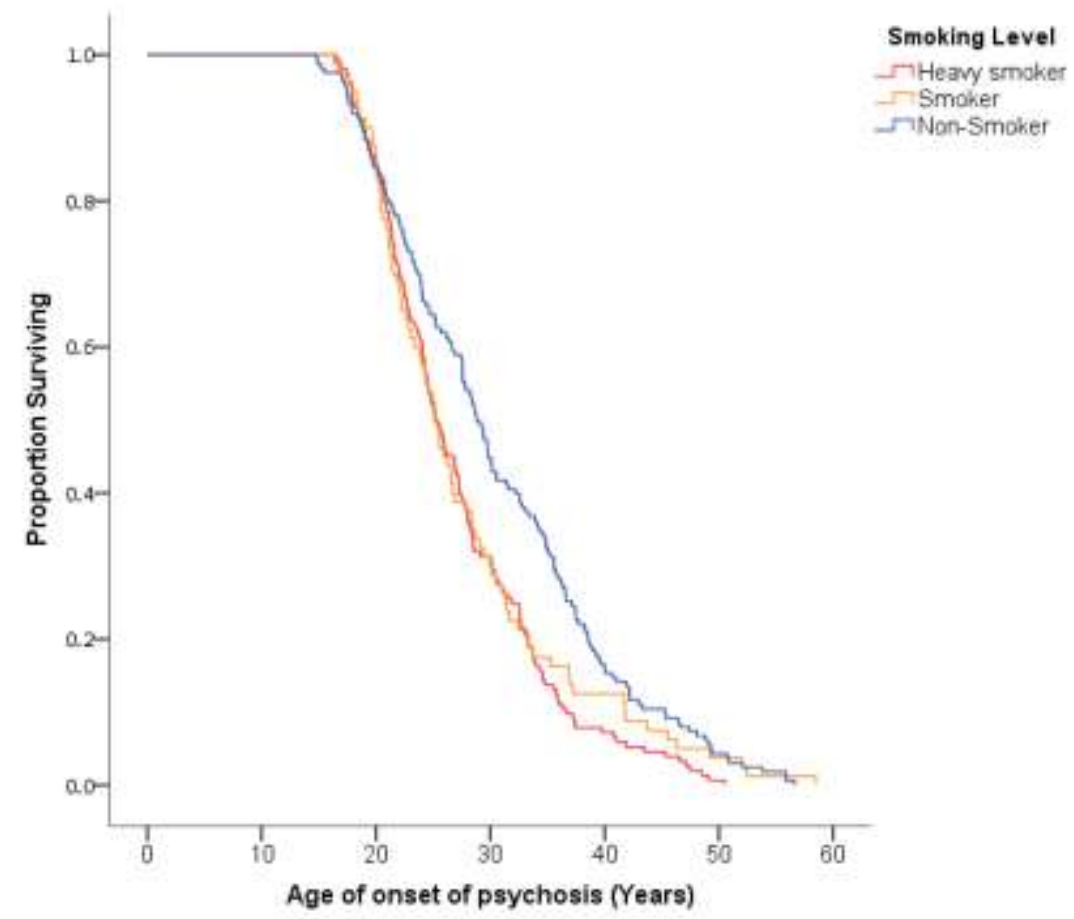

a Kaplan Meier survival curve. Proportion surviving refers to the proportion of patients not yet having a diagnosis of a psychotic illness. 
Figure 3: Cumulative survival curve of smoking status and age at onset of psychosis. ${ }^{a}$

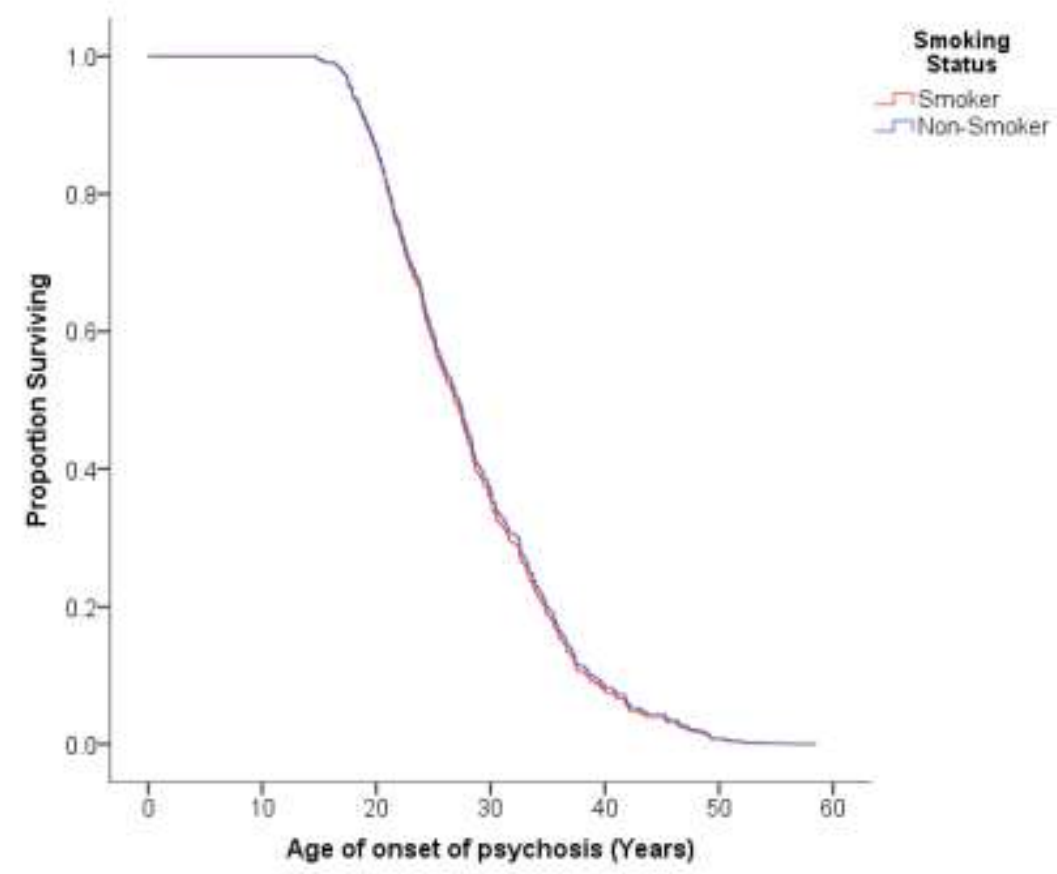

${ }^{a}$ Cox Proportional Hazard model, with cannabis use (yes/no), family history of psychosis (yes/no) and sex as covariates. Proportion surviving refers to the proportion of patients not yet having a diagnosis of a psychotic illness. 
Table 1: Clinical and demographic characteristics of patients, stratified by level of tobacco use.

\begin{tabular}{|c|c|c|c|c|c|c|c|c|}
\hline & \multicolumn{2}{|c|}{$\begin{array}{c}\text { All } \\
(\mathrm{N}=397)\end{array}$} & \multicolumn{2}{|c|}{$\begin{array}{c}\text { Non-smoker } \\
(\mathrm{N}=164)\end{array}$} & \multicolumn{2}{|c|}{$\begin{array}{l}\text { Smoker } \\
(\mathrm{N}=233)\end{array}$} & \multicolumn{2}{|c|}{$\begin{array}{l}\text { Non-smoker vs } \\
\text { Smoker }\end{array}$} \\
\hline & Mean & SD & Mean & SD & Mean & SD & $t(d f=1)$ & $p$ \\
\hline Age, yrs & 29.8 & 9.5 & 32.2 & 10.5 & 28.2 & 8.3 & -4.002 & $<0.001$ \\
\hline Age at psychosis onset, yrs & 28.7 & 9.0 & 30.5 & 9.9 & 27.4 & 8.1 & 3.306 & 0.001 \\
\hline Age at smoking onset, $\mathrm{yrs}^{\mathrm{a}}$ & 15.9 & 3.0 & - & - & 15.9 & 3.0 & - & - \\
\hline DUI,months ${ }^{\mathrm{b}}$ & 25.3 & 40.6 & 30.6 & 52.6 & 21.7 & 29.4 & 1.921 & 0.056 \\
\hline DUP, months ${ }^{c}$ & 13.8 & 30.8 & 19.2 & 42.2 & 10.0 & 18.2 & 2.609 & 0.010 \\
\hline Total SAPS at baseline & 13.5 & 4.3 & 13.2 & 4.31 & 13.7 & 4.36. & 1.235 & 0.217 \\
\hline \multirow[t]{2}{*}{ Total SANS at baseline } & 7.1 & 6.3 & 6.68 & 6.04 & 7.3 & 4 & 1.04 & 0.299 \\
\hline & $\mathrm{N}$ & $\%$ & $\mathrm{~N}$ & $\%$ & $\mathrm{~N}$ & $\%$ & $\begin{array}{c}x^{2} \\
(d f=1)\end{array}$ & $p$ \\
\hline Sex (Male) & 226 & 56.9 & 75 & 45.7 & 151 & 64.8 & 14.283 & $<0.001$ \\
\hline Diagnosis & & & & & & & & \\
\hline Schizophrenia & 274 & 69.0 & 106 & 64.6 & 168 & 72.1 & 2.518 & 0.284 \\
\hline $\begin{array}{l}\text { Other primary } \\
\text { psychotic disorder }\end{array}$ & 123 & 31.0 & 58 & 35.4 & 65 & 27.9 & & \\
\hline Unemployed $(Y e s)^{d}$ & 176 & 44.3 & 6324 & 38.4 & 113 & 48.7 & 4.122 & 0.042 \\
\hline Cannabis Use (Yes) & 171 & 43.1 & & 14.6 & 147 & 63.1 & 92.168 & $<0.001$ \\
\hline
\end{tabular}

This article is protected by copyright. All rights reserved. 
Table 2: Adjusted and unadjusted hazard ratios for the association between age of onset of psychosis and tobacco use.

\begin{tabular}{l|c|c|c} 
& Hazard Ratio & 95\% Confidence Interval & $\mathrm{p}$ \\
\hline Unadjusted & 0.703 & $0.574-0.861$ & $\mathbf{0 . 0 0 1}$ \\
Adjusted for sex & 2.062 & $1.669-2.548$ & $<0.001$ \\
Adjusted for cannabis use & 2.451 & $1.982-3.031$ & $<\mathbf{0 . 0 0 1}$ \\
Adjusted for family history of & 0.973 & $0.766-1.235$ & 0.820 \\
psychosis & & & \\
Adjusted for all covariates & 1.034 & $0.828-1.291$ & 0.767 \\
\hline
\end{tabular}

